\title{
Scanning TEM Electron Beam Induced Current Imaging in Water
}

Jared J. Lodico ${ }^{1}$, William A. Hubbard ${ }^{1}$ and B. C. Regan ${ }^{1}$

${ }^{1 .}$ Department of Physics \& Astronomy and California NanoSystems Institute, University of California, Los Angeles, CA.

Despite its key role in determining the properties of wet chemical systems, the electrical double layer is not well understood [1,2]. Direct observation of the EDL requires a high resolution imaging technique that is also compatible with in situ wet cell experimentation. Transmission electron microscopy (TEM) represents the gold-standard for determining structures at molecular length scales and, in recent years, has been used regularly to probe liquid systems [3]. Traditional TEM signals, however, mostly probe physical structure and are largely insensitive to the electronic structure of a material. Here we use electron beam induced current (EBIC) imaging, traditionally used to characterize semiconductor devices with intrinsic electric fields $[4,5]$, in a scanning TEM (STEM) to image the electron-hole pair separation in a water fluid cell under bias in an effort more completely understand the electronic structure of liquids.

The in situ fluid cells consist of two $\mathrm{Si} / \mathrm{SiO}_{2}$ chips, each with an electron-transparent $\mathrm{Si}_{3} \mathrm{~N}_{4}$ window, sandwiched together. $\mathrm{Ti} / \mathrm{Pt}(5 / 25 \mathrm{~nm})$ electrodes are patterned onto one of the chips via optical lithography. To form a wet cell, a small droplet of deionized water is placed on the patterned chip. The second, smaller chip is placed on top of the droplet and manipulated to align the two windows, sandwiching a thin layer of water in between. Epoxy is then used to glue the two chips together creating a vacuum-compatible fluid cell. We use a Hummingbird Scientific biasing holder to electrically contact the fluid cell, and we image the sample in an FEI Titan 80-300 S/TEM at $300 \mathrm{kV}$ accelerating voltage. Figure 1a and $\mathrm{b}$ show bright field (BF) and annular dark field (ADF) images of an electrode in an assembled water fluid cell.

As the electron beam scans the sample the current induced, either via electron-hole pair separation or secondary electrons generation, is collected at each pixel via transimpedance current amplifiers attached to electrodes within the wet cell. Dark (bright) contrast is generated when electrons (holes) pass through an electrode into the transimpedance current amplifier. Figure 1c and d, acquired simultaneously with Fig. 1a and b, show STEM EBIC images of an electrode under $1 \mathrm{~V}$ bias, and Fig. $1 \mathrm{~g}$ and $\mathrm{h}$ show the same device under $-1 \mathrm{~V}$ bias. The image in Fig. 1d is generated by collection of current in the electrode being imaged/biased (red current meter symbol), while the Fig. 1c image shows current collected in an electrode just barely within the field of view (blue current meter symbol), which is held at virtual ground. As an electron-hole pair is generated over the electrode the electrons are attracted to the positively charged electrode, generating a dark signal in Fig. 1d, and the holes travel several microns through the water to the electrode held at virtual ground, producing an equal and opposite current seen in Fig. 1c. The effect is reversed in a negative bias polarity, as shown in Fig. $1 \mathrm{~g}$ and $\mathrm{h}$. The magnitude of the current measured on the electrode, relative to the background current, is a few $\mathrm{pA}$ in all four EBIC images [6]. 
References:

[1] P. Attard, Advances in Chemical Physics 92 (1996), p. 1.

[2] M. S. Kilic et al, Physical Review E 75 (2007) p. 021502.

[3] M. J. Williamson et al, Nature Materials 2 (2003), p. 532.

[4] H. Leamy, J. Appl. Phys. 53 (1982) p. R51.

[5] E. R. White et al, Applied Physics Letters 107 (2015), p. 223104.

[6] This work was supported by National Science Foundation (NSF) award DMR-1611036, and by NSF

STC award DMR-1548924.

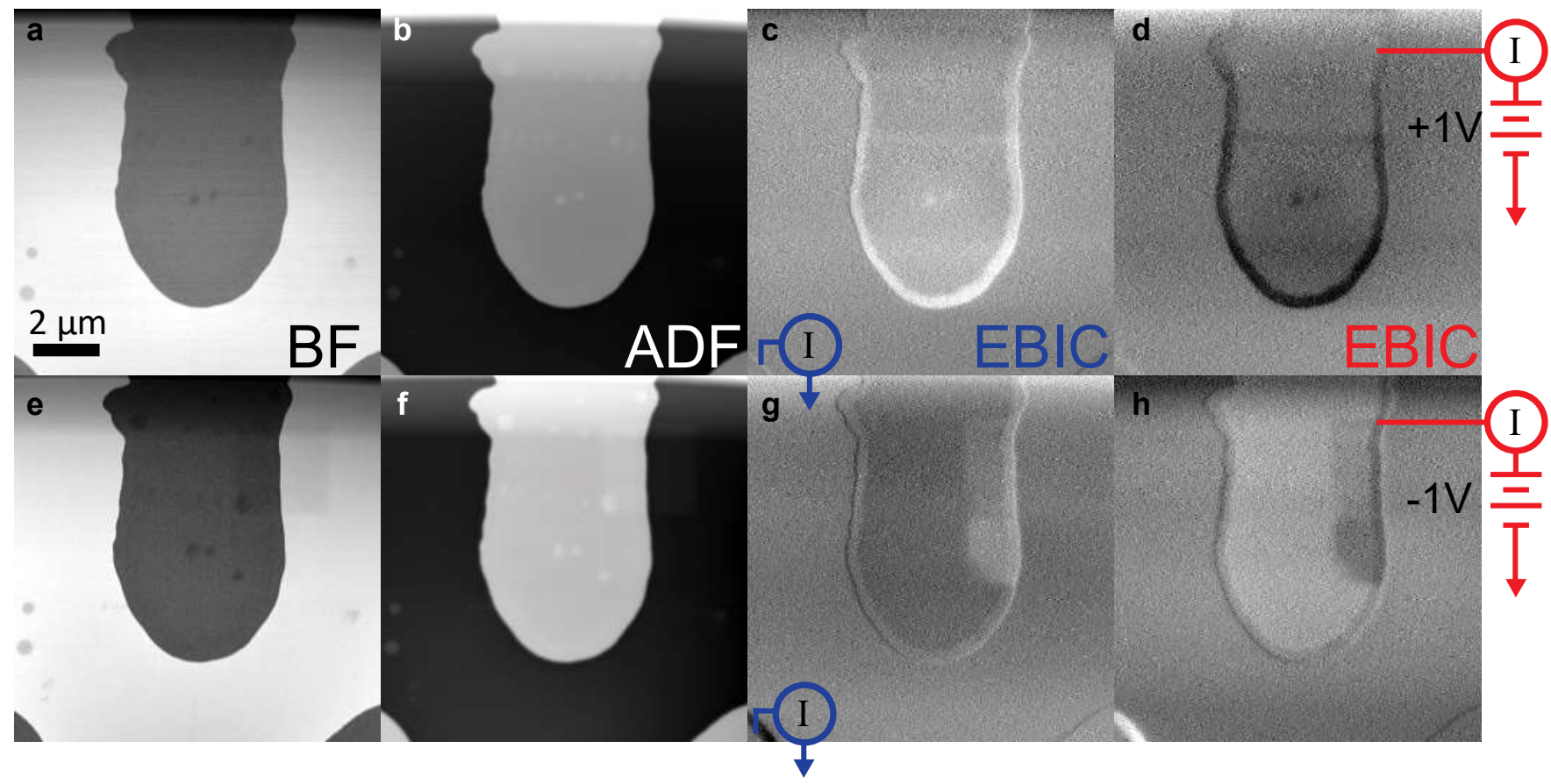

Figure 1. Bright field (a, e), angular dark field (b, f), and electron beam induced current (c, d, g, h) images, acquired simultaneously, showing a Ti/ $\mathrm{Pt}(5 / 25 \mathrm{~nm})$ electrode in a water fluid cell. The images in (c) and (g) are generated by collecting current from an electrode nearly out of the field of view (blue current meter symbol). In (d) and (h), current is measured from the electrode featured most prominently in the images (red current meter symbol). The electrode is biased to $1 \mathrm{~V}$ in (a)-(d) and -1 V in (e)-(h). 